\title{
Study of a Newtonian Fluid through Circular Channels with Slip Boundary Taking into Account Electrokinetic Effect
}

\author{
Qian Sun, ${ }^{1}$ Yonghong $\mathrm{Wu},{ }^{1}{ }^{\text {Lishan Liu, }}{ }^{2}$ and B. Wiwatanapataphee ${ }^{3}$ \\ ${ }^{1}$ Department of Mathematics and Statistics, Curtin University, Perth, WA 6845, Australia \\ ${ }^{2}$ School of Mathematical Sciences, Qufu Normal University, Qufu, Shandong 273165, China \\ ${ }^{3}$ Department of Mathematics, Faculty of Science, Mahidol University, Bangkok 10400, Thailand
}

Correspondence should be addressed to Qian Sun; julysunqian@gmail.com and

B. Wiwatanapataphee; benchawan.wiw@mahidol.ac.th

Received 8 September 2013; Accepted 8 October 2013

Academic Editor: Shaoyong Lai

Copyright (C) 2013 Qian Sun et al. This is an open access article distributed under the Creative Commons Attribution License, which permits unrestricted use, distribution, and reproduction in any medium, provided the original work is properly cited.

\begin{abstract}
We study the slip flow of fluids driven by the combined effect of electrical force and pressure gradient. The underlying boundary value problem is solved through the use of Fourier series expansion in time and Bessel function in space. The exact solutions and numerical investigations show that the slip length and electrical field parameters have significant effects on the velocity profile. By varying these system parameters, one can achieve smooth velocity profiles or wave form profiles with different wave amplitude and frequency. This opens the way for optimizing the flow by choosing the slip length, the electrical field, and electrolyte solutions.
\end{abstract}

\section{Introduction}

Over the past few decades, advances in nanoscience and nanotechnology have led to the development of many microelectromechanical systems and devices such as heat exchanger [1], micropump [2], lab-on-a-chip diagnostic devises [3], drug delivery systems [4], energy conversion, and biological sensing devices [5]. Most of these systems and devices involve fluid flow in microtubes and microchannels. To control the microfluidics in microchannels so as to achieve optional system performance, it is essential to study the fundamental mechanics of microflows and derive better models and understanding of the flow mechanism and flow behaviour.

Flow of microfluidics may be driven by pressure gradient or by electrical forces. For electrical-driven flow, the solid surface of the microchannel is electrically charged to generate a region in the fluid with a distribution of electrical charges near the channel surface. This region is called the electrical double layer (EDL). The EDL, on one hand, has the effect of retarding liquid flow driven by external pressure gradient to form a streaming potential, whereas, on the other hand, it can induce fluid flow by applying an external electric field. In this work, we will study the flow of microfluidics in microchannels driven by the combination of pressure gradient and externally applied electric field.

The equations governing the flow of microfluidics in microchannels include the Navier-Stokes equations, the incompressible continuity equation, and boundary conditions. Traditionally, the no-slip boundary condition is used $[6,7]$. However, recent molecular dynamic simulations and experiments in micrometer scale show that the flow of fluids in micrometer scale is granular and slip occurs between the fluid and the solid surface [8-12]. Therefore, the no-slip condition does not work for fluid flow in microchannels. In this work, the Navier slip boundary condition will be used; namely, the tangential fluid velocity relative to the solid surface is proportional to the shear stress on the solid-fluid interface. The validity of the Navier slip boundary condition is supported by many experimental results [13-15].

For many fluid flow problems, under the nonslip assumption, exact and numerical solutions have been obtained and can be found in the literature [6, 16-18]. Steady state solutions under slip conditions have also been established for flows of Newtonian fluids through pipes, channels, and annulus $[19,20]$. More recently, various analytical solutions 
for pressure-driven time-dependent slip flows of Newtonian fluids through microtubes and microannulus were derived $[16,18]$. Various attempts have also been made to study the electrically driven fluid flows. Rice and Whitehead [21] investigated the steady-state liquid flow due to an electric field in circular capillaries. Levine et al. [22] analysed the electrokinetic steady flow in a narrow parallel-plate microchannel. Yang et al. [23] studied the flow in rectangular microchannels and Mala et al. [24] in parallel-plate microchannels.

Motivated by the previous work, this work aims to generalize the result in [25] for the pressure-driven slip flow to the case with the combined effect of pressure-driving forces and electrically driving forces. The rest of the paper is organized as follows. In Section 2, we present the mathematical model for the problem, consisting of the governing field equations and boundary conditions. In Section 3, we derive the exact solutions for the velocity field through the use of Bessel functions in space and Fourier series expansion in time. Based on the velocity solutions, we then establish the analytical solutions for the stress tensor and flow rate. In Section 5, we investigate the influence of the electric field and the surface slippage on the flow behaviour. Then a conclusion is given in Section 6.

\section{Mathematical Model and Formulation}

In this paper, we consider the flow of microfludics through a circular microchannel driven by both pressure gradient and external electric field. The cylindrical polar coordinate $(r, \theta, z)$, with the $z$-axis being in the axial direction, is used in the formulation. The governing field equations for the problem include the Navier-Stokes equations and the continuity equation. Let $\underline{v}=\left(v_{r}, v_{\theta}, u\right)$ be the velocity vector with $v_{r}, v_{\theta}$, and $u$ being, respectively, the components of velocity in the radial direction, the transverse direction, and axial direction. Then the continuity equation and the NavierStokes equation in the $z$-direction are

$$
\begin{gathered}
\frac{\partial^{2} v_{r}}{\partial r^{2}}+\frac{v_{r}}{r}+\frac{\partial v_{\theta}}{r \partial \theta}+\frac{\partial u}{\partial z}=0 \\
\frac{\partial u}{\partial t}+v_{r} \frac{\partial u}{\partial r}+\frac{v_{\theta}}{r} \frac{\partial u}{\partial \theta}+u \frac{\partial u}{\partial \theta} \\
=g_{z}-\frac{1}{\rho_{f}} \frac{\partial p}{\partial z} \\
+\frac{\mu}{\rho_{f}}\left(\frac{\partial^{2} u}{\partial r^{2}}+\frac{\partial u}{r \partial r}+\frac{1}{r^{2}} \frac{\partial^{2} u}{\partial \theta^{2}}+\frac{\partial^{2} u}{\partial z^{2}}\right)+\frac{\rho_{e}}{\rho_{f}} E_{z}^{\prime},
\end{gathered}
$$

where $p$ is the pressure, $\mu$ is the dynamic viscosity, $\rho_{f}$ and $\rho_{e}$, are respectively, the fluid density and the electric charge density, and $E_{z}^{\prime}$ is the externally applied electric field.

Assuming that the flow is axially symmetric and the radial and transverse velocity components are negligible, then (1) admits solution of the form

$$
v_{r}=0, \quad v_{\theta}=0, \quad u=u(r, t)
$$

with $u(r, t)$ determined by

$$
\frac{\partial u}{\partial t}-\frac{\mu}{\rho_{f}}\left(\frac{\partial^{2} u}{\partial r^{2}}+\frac{1}{r} \frac{\partial u}{\partial r}\right)=-\frac{1}{\rho_{f}} \frac{\partial p}{\partial z}-\frac{\rho_{e}}{\rho_{f}} \vec{E}_{z}^{\prime} .
$$

Based on [25], the velocity boundary condition for the problem can be written as

$$
\frac{\partial u}{\partial r}(0, t)=0, \quad u(R, t)=-l \frac{\partial u}{\partial r}(R, t) .
$$

In this paper, we study the flow driven by both pressure gradient $\partial p / \partial z$ and an externally applied electrical field $E_{z}^{\prime}$. Without loss of generality, we express the pressure gradient by Fourier series; namely,

$$
\frac{\partial p}{\partial z}=\bar{q}(t)=a_{0}+\sum_{n=1}^{\infty}\left[a_{n} \cos (n \omega t)+b_{n} \sin (n \omega t)\right],
$$

which can also be expressed by

$$
\frac{\partial p}{\partial z}=\operatorname{Re}\left(\sum_{n=0}^{\infty} c_{n} e^{i n \omega t}\right)
$$

where $\operatorname{Re}()$ is to take the real part of the complex quantity. To determine the free charge density $\rho_{e}$, let $\psi(r)$ be the electric potential associated with double layer at the equilibrium state. Then based on [26], we have

$$
\begin{gathered}
\rho_{e}=-\kappa^{2} \psi, \\
\frac{d^{2} \psi}{d r^{2}}+\frac{1}{r} \frac{d \psi}{d r}=\kappa^{2} \psi, \\
\psi(R)=\psi_{s}, \quad \psi(0) \text { is finite, }
\end{gathered}
$$

where $\psi_{s}$ is the surface potential at the wall $r=R$. Now substituting (7) into (3), we have

$$
\frac{\partial u}{\partial t}-\frac{\mu}{\rho_{f}}\left(\frac{\partial^{2} u}{\partial r^{2}}+\frac{1}{r} \frac{\partial u}{\partial r}\right)=-\frac{1}{\rho_{f}} \frac{\partial p}{\partial z}+\frac{1}{\rho_{f}} \kappa^{2} \psi E_{z}^{\prime} .
$$

Hence the problem is to solve the boundary value problem (8)-(9) for $\psi$ and then the partial differential equation (10) subject to boundary conditions (4) for $u$, which will be done in Section 3.

\section{Solution for the Transient Velocity Field}

Let $\kappa r=\bar{r}$; (8) becomes

$$
\bar{r}^{2} \frac{d^{2} \psi}{d \bar{r}^{2}}+\bar{r} \frac{d \psi}{d \bar{r}}-\bar{r}^{2} \psi=0
$$

which gives solution

$$
\psi=A J_{0}(\kappa r)+B Y_{0}(\kappa r)
$$

where $A$ and $B$ denote integration constants and $J_{0}$ and $Y_{0}$ are the zero-order Bessel functions of the first and second kinds, respectively. From (12) and boundary condition (9), we obtain

$$
\psi=\frac{\psi_{s}}{J_{0}(\kappa R)} J_{0}(\kappa r)
$$


and (10) becomes

$$
\begin{aligned}
\frac{\partial u}{\partial t} & -\frac{\mu}{\rho_{f}}\left(\frac{\partial^{2} u}{\partial r^{2}}+\frac{1}{r} \frac{\partial u}{\partial r}\right) \\
& =-\frac{1}{\rho_{f}} \operatorname{Re} \sum_{n=1}^{\infty} c_{n} e^{i n \omega t}+\frac{1}{\rho_{f}} \frac{\kappa^{2} \psi_{s}}{J_{0}(\kappa R)} J_{0}(\kappa r) E_{z}^{\prime}
\end{aligned}
$$

which admits solution of the form

$$
u(r, t)=u_{p}(r, t)+u_{e}(r, t),
$$

where $u_{p}$ is the solution corresponding to the first term on the right hand side and $u_{e}$ is the solution contributed from the second term of the right hand side. From the superposition principle, based on the work of [25], we have

$$
\begin{aligned}
u_{p}(r, t)= & C+\frac{a_{0}}{4 \mu} r^{2} \\
& +\sum_{n=1}^{\infty} \operatorname{Re}\left\{\left(d_{n} J_{0}\left(\beta_{n} r\right)+\frac{c_{n} i}{\rho_{f} n \omega}\right) e^{i n \omega t}\right\},
\end{aligned}
$$

where $\beta_{n}^{2}=n \beta^{2}$ with $\beta^{2}=\left(\rho_{f} \omega / \mu\right) i$. Now, we proceed to find the solution contributed from the second term on the right hand side of (15). Let $u_{e}(r)=C_{0}(r) t+C_{1}(r)$ and substitute it into (14); then we have

$$
\begin{aligned}
-\frac{\mu}{\rho_{f}} & {\left[C_{0}^{\prime \prime}(r)+\frac{1}{r} C_{0}^{\prime}(r)\right] t+C_{0}(r)-\frac{\mu}{\rho_{f}}\left[C_{1}^{\prime \prime}(r)+\frac{1}{r} C_{1}^{\prime}(r)\right] } \\
& =\frac{1}{\rho_{f}} \frac{\kappa^{2} \psi_{s}}{J_{0}(\kappa R)} E_{z}^{\prime} J_{0}(\kappa r) .
\end{aligned}
$$

For the above equation to hold for any instant of time $t$, we require

$$
\begin{gathered}
C_{0}^{\prime \prime}(r)+\frac{1}{r} C_{0}^{\prime}(r)=0, \\
C_{0}(r)-\frac{\mu}{\rho_{f}}\left[C_{1}^{\prime \prime}(r)+\frac{1}{r} C_{1}^{\prime}(r)\right] \\
=\frac{1}{\rho_{f}} \frac{\kappa^{2} \psi_{s}}{J_{0}(\kappa R)} E_{z}^{\prime} J_{0}(\kappa r) .
\end{gathered}
$$

Thus,

$$
C_{0}=D_{1} \ln r+D_{2} \text {. }
$$

As $C_{0}$ must be bounded at $r=0$ but $\ln r$ has singularity at $r=0$, we require that $D_{1}=0$. From (18), we have

$$
C_{1}=\frac{1}{\mu} \frac{\psi_{s} E_{z}^{\prime}}{J_{0}(\kappa R)} J_{0}(\kappa r)+\frac{\rho_{f}}{\mu} \frac{D_{2}}{4} r^{2},
$$

and then by substituting $C_{0}$ and $C_{1}$ into $u_{e}(r, t)$, we have

$$
u_{e}(r, t)=D_{2} t+\frac{1}{\mu} \frac{\psi_{s} E_{z}^{\prime}}{J_{0}(\kappa R)} J_{0}(\kappa r)+\frac{\rho_{f}}{\mu} \frac{D_{2}}{4} r^{2},
$$

Hence, by substituting (16) and (21) into (15), we obtain

$$
\begin{aligned}
u(r, t)= & C+\frac{a_{0}}{4 \mu} r^{2} \\
& +\sum_{n=1}^{\infty} \operatorname{Re}\left\{\left(d_{n} J_{0}\left(\beta_{n} r\right)+\frac{c_{n} i}{\rho_{f} n \omega}\right) e^{i n \omega t}\right\} \\
& +D_{2} t+\frac{1}{\mu} \frac{\psi_{s} E_{z}^{\prime}}{J_{0}(\kappa R)} J_{0}(\kappa r)+\frac{\rho_{f}}{\mu} \frac{D_{2}}{4} r^{2} .
\end{aligned}
$$

Substituting (22) into boundary condition (4) yields

$$
\begin{aligned}
C+ & \frac{a_{0}}{4 \mu} R^{2}+l \frac{a_{0}}{2 \mu} R+\frac{1}{\mu} \psi_{s} E_{z}^{\prime}-l \frac{\kappa \psi_{s} E_{z}^{\prime}}{\mu J_{0}(\kappa R)} J_{1}(\kappa R) \\
& +D_{2} t+\frac{\rho_{f} D_{2} R^{2}}{4 \mu}+l \frac{\rho_{f} D_{2} R}{2 \mu} \\
& +\sum_{n=1}^{\infty} \operatorname{Re}\left[\left(d_{n} J_{0}(\beta R)+\frac{c_{n} i}{\rho_{f} n \omega}-l \beta_{n} d_{n} J_{1}\left(\beta_{n} R\right)\right) e^{i n \omega t}\right]
\end{aligned}
$$$$
=0 .
$$

For the above equation to hold for any instant of time $t$, we require $D_{2}=0$ and

$$
\begin{gathered}
C+\frac{a_{0}}{4 \mu} R^{2}+l \frac{a_{0}}{2 \mu} R+\frac{1}{\mu} \psi_{s} E_{z}^{\prime} \\
-l \frac{\kappa}{\mu} \frac{\psi_{s} E_{z}^{\prime}}{J_{0}(\kappa R)} J_{1}(\kappa R)=0, \\
d_{n} J_{0}(\beta R)+\frac{c_{n} i}{\rho_{f} n \omega}-l \beta_{n} d_{n} J_{1}\left(\beta_{n} R\right)=0,
\end{gathered}
$$

which give

$$
\begin{gathered}
C=-\frac{a_{0} R^{2}}{4 \mu}\left(1+\frac{2 l}{R}\right)-\frac{1}{\mu} \psi_{s} E_{z}^{\prime}+l \frac{\kappa}{\mu} \frac{\psi_{s} E_{z}^{\prime}}{J_{0}(\kappa R)} J_{1}(\kappa R), \\
d_{n}=\frac{-c_{n} i}{\rho_{f} n \omega\left[J_{0}(\beta R)-l \beta_{n} J_{1}\left(\beta_{n} R\right)\right]} .
\end{gathered}
$$

Hence, by substituting (25) into (22), we obtain

$$
\begin{aligned}
u= & -\frac{a_{0} R^{2}}{4 \mu}\left(1+\frac{2 l}{R}-\frac{r^{2}}{R^{2}}\right)-\frac{1}{\mu} \psi_{s} E_{z}^{\prime} \\
& +l \frac{\kappa}{\mu} \frac{\psi_{s} E_{z}^{\prime}}{J_{0}(\kappa R)} J_{1}(\kappa R) \\
& -\sum_{n=1}^{\infty} \operatorname{Re}\left\{\frac{c_{n} i}{\rho_{f} n \omega}\left(\frac{J_{0}\left(\beta_{n} r\right)}{J_{0}\left(\beta_{n} R\right)-l \beta_{n} J_{1}\left(\beta_{n} R\right)}-1\right) e^{i n \omega t}\right\} \\
& +\frac{1}{\mu} \frac{\psi_{s} E_{z}^{\prime}}{J_{0}(\kappa R)} J_{0}(\kappa r) .
\end{aligned}
$$


We should remark that if $l=0$, solution (26) reduces to the no-slip solution

$$
\begin{aligned}
u= & -\frac{a_{0} R^{2}}{4 \mu}\left(1-\frac{r^{2}}{R^{2}}\right)-\frac{1}{\mu} \psi_{s} E_{z}^{\prime} \\
& -\sum_{n=1}^{\infty} \operatorname{Re}\left\{\frac{c_{n} i}{\rho_{f} n \omega}\left(\frac{J_{0}\left(\beta_{n} r\right)}{J_{0}\left(\beta_{n} R\right)}-1\right) e^{i n \omega t}\right\} \\
& +\frac{1}{\mu} \frac{\psi_{s} E_{z}^{\prime}}{J_{0}(\kappa R)} J_{0}(\kappa r) .
\end{aligned}
$$

\section{Solutions for the Flow Rate and Stresses}

From the velocity solution (26), we get the flow rate as follows:

$$
\begin{aligned}
& Q(t)= \int_{0}^{R} 2 \pi r u d r \\
&=-\frac{a_{0} \pi R^{3}}{2 \mu}\left(l+\frac{R}{4}\right)+l \frac{\pi R^{2} \kappa}{\mu} \frac{\psi_{s} E_{z}^{\prime}}{J_{0}(\kappa R)} J_{1}(\kappa R) \\
&+\int_{0}^{R} \frac{1}{\mu} \frac{\psi_{s} E_{z}^{\prime}}{J_{0}(\kappa R)} J_{0}(\kappa r) r d r-\frac{2 \pi}{\rho_{f} \omega} \\
& \times \operatorname{Re}\left[\sum _ { n = 1 } ^ { \infty } \frac { c _ { n } i e ^ { i n \omega t } } { n } \int _ { 0 } ^ { R } \left(\frac{J_{0}\left(\beta_{n} r\right)}{J_{0}\left(\beta_{n} R\right)-l J_{1}\left(\beta_{n} R\right)}\right.\right. \\
&-1) r d r]-\frac{\pi R^{2}}{\mu} \psi_{s} E_{z}^{\prime} .
\end{aligned}
$$

From the identity

$$
\frac{d}{d x}\left[x^{n} J_{n}(x)\right]=x^{n} J_{n-1}(x),
$$

we have

$$
\frac{d}{d r}\left[r J_{1}\left(\beta_{n} r\right)\right]=\beta_{n} r J_{0}\left(\beta_{n} r\right)
$$

and hence

$$
\int_{0}^{R} r J_{0}\left(\beta_{n} r\right) d r=\frac{1}{\beta_{n}}\left[r J_{1}\left(\beta_{n} r\right)\right]_{0}^{R}=\frac{1}{\beta_{n}} R J_{1}\left(\beta_{n} R\right) .
$$

Therefore from (28) and the above formula, we get

$$
\begin{aligned}
& Q(t)=-\frac{a_{0} \pi R^{3}}{2 \mu}\left(l+\frac{R}{4}\right)+l \frac{\pi R^{2} \kappa}{\mu} \frac{\psi_{s} E_{z}^{\prime}}{J_{0}(\kappa R)} J_{1}(\kappa R) \\
&+\frac{R}{\mu \kappa} \frac{\psi_{s} E_{z}^{\prime}}{J_{0}(\kappa R)} J_{1}(\kappa R)-\frac{2 \pi}{\rho_{f} \omega} \\
& \times \operatorname{Re}\left[\sum _ { n = 1 } ^ { \infty } \frac { c _ { n } i e ^ { i n \omega t } } { n } \left(\frac{R J_{1}\left(\beta_{n} R\right)}{\beta_{n} J_{0}\left(\beta_{n} R\right)-l \beta_{n} J_{1}\left(\beta_{n} R\right)}\right.\right. \\
&\left.\left.-\frac{R^{2}}{2}\right)\right]-\frac{\pi R^{2}}{\mu} \psi_{s} E_{z}^{\prime} .
\end{aligned}
$$

The total amount of fluid, flowing through the tube during the period $[0, T]$, can then be determined as follows:

$$
\begin{aligned}
Q_{T}= & \int_{0}^{T} Q(t) d t \\
= & -\frac{a_{0} \pi R^{3} T}{2 \mu}\left(l+\frac{R}{4}\right)+l \frac{\pi R^{2} \kappa}{\mu} \frac{\psi_{s} E_{z}^{\prime} T}{J_{0}(\kappa R)} J_{1}(\kappa R) \\
& +\frac{\pi R}{\mu \kappa} \frac{\psi_{s} E_{z}^{\prime} T}{J_{0}(\kappa R)} J_{1}(\kappa R)-\frac{2 \pi}{\rho_{f} \omega^{2}} \\
& \times \operatorname{Re}\left[\sum_{n=1}^{\infty} \frac{c_{n}\left(e^{i n \omega T}-1\right)}{n^{2}}\right. \\
& -\frac{\pi R^{2}}{\mu} \psi_{s} E_{z}^{\prime} T .
\end{aligned}
$$

Now, we determine the stresses in the fluid. From $v=$ $e_{r} 0+e_{\theta} 0+e_{z} u(r)$, we get

$$
\nabla v=\left(\begin{array}{ccc}
0 & 0 & \frac{\partial u}{\partial r} \\
0 & 0 & 0 \\
\frac{\partial u}{\partial r} & 0 & 0
\end{array}\right)
$$

From the above formulae and (26), we obtain

$$
\begin{gathered}
d_{r \theta}=d_{\theta z}=d_{r r}=d_{\theta \theta}=d_{z z}, \\
d_{r z}=\frac{a_{0} r}{2 \mu}+\sum_{n=1}^{\infty} \operatorname{Re}\left[\frac{c_{n} i}{\rho_{f} n \omega}\left(\frac{\beta_{n} J_{1}\left(\beta_{n} r\right)}{J_{0}\left(\beta_{n} R\right)-l \beta_{n} J_{1}\left(\beta_{n} R\right)}\right) e^{i n \omega t}\right] \\
-\frac{\kappa}{\mu} \frac{\psi_{s} E_{z}^{\prime}}{J_{0}(\kappa R)} J_{1}(\kappa r) .
\end{gathered}
$$

Thus from the constitutive equations for Newtonian fluid, we get

$$
\begin{gathered}
\sigma_{r r}=\sigma_{\theta \theta}=\sigma_{z z}=-p=\bar{q}(t) x+p_{0}(t), \\
\sigma_{r \theta}=\sigma_{\theta z}=0 \\
\sigma_{r z}=a_{0} r \\
+2 \mu \sum_{n=1}^{\infty} \operatorname{Re}\left[\frac{c_{n} i}{\rho_{f} n \omega}\left(\frac{\beta_{n} J_{1}\left(\beta_{n} r\right)}{J_{0}\left(\beta_{n} R\right)-l \beta_{n} J_{1}\left(\beta_{n} R\right)}\right) e^{i n \omega t}\right] \\
-2 \mu \kappa \frac{\psi_{s} E_{z}^{\prime}}{J_{0}(\kappa R)} J_{1}(\kappa r),
\end{gathered}
$$

where $p_{0}(t)$ is an arbitrary constant which may be chosen to meet certain pressure conditions. 


\section{Numerical Investigation}

In this section, we use the solutions obtained in previous sections to study the influence of the combined effect of electrical field and pressure gradient on the velocity profile and flow rate. As the pressure gradient can be expressed as a Fourier series, without loss of generality, we consider here only two cases of pressure gradient, including the constant pressure gradient and a cosine wave form pressure gradient.

Case 1. Consider

$$
\frac{d p}{d z}=a_{0}
$$

In this case, we have $c_{n}=0$ for all $n \geq 1$, and thus, from (26) and (32), we get the normalized velocity and flow rate as follows:

$$
\begin{aligned}
u^{*}= & -\frac{4 \mu}{a_{0} R^{2}} u \\
= & \left(1+\frac{2 l}{R}-\frac{r^{2}}{R^{2}}\right) \\
& -\frac{4 \mu}{a_{0} R^{2}}\left[l \frac{\kappa}{\mu} \frac{J_{1}(\kappa R)}{J_{0}(\kappa R)}+\frac{1}{\mu} \frac{J_{0}(\kappa r)}{J_{0}(\kappa R)}-\frac{1}{\mu}\right] \psi_{s} E_{z}^{\prime}, \\
Q^{*}(t)= & -\frac{2 \mu}{a_{0} \pi R^{3}} Q(t) \\
= & \left(l+\frac{R}{4}\right)-\frac{2 \mu}{a_{0} \pi R^{2}} \\
& \times\left[l \frac{\pi \kappa R}{\mu} \frac{J_{1}(\kappa R)}{J_{0}(\kappa R)}+\frac{1}{\mu \kappa} \frac{J_{1}(\kappa R)}{J_{0}(\kappa R)}-\frac{\pi R}{\mu}\right] \psi_{s} E_{z}^{\prime} .
\end{aligned}
$$

We should address here that the velocity and flow rate are linearly propositional to the slip length $l$.

Case 2. Consider

$$
\frac{d p}{d z}=a_{1} \cos (\omega t)
$$

In this case, we have $a_{0}=0, c_{1}=a_{1} \in R, c_{n}=0$ for all $n \geq 2$. For simplification, we normalize the variables as follows:

$$
\begin{array}{ll}
\beta^{*}=\beta R, & r^{*}=\frac{r}{R} \in[0,1], \quad l^{*}=\frac{l}{R}, \\
t^{*}=\frac{\omega t}{2 \pi}, & u^{*}=-\frac{\rho_{f} \omega}{a_{1}} u,
\end{array}
$$

Then from (26), we get

$$
\begin{aligned}
u^{*}= & \operatorname{Re}\left(\left(\frac{J_{0}\left(\beta^{*} r^{*}\right)}{J_{0}\left(\beta^{*}\right)-l^{*} \beta^{*} J_{1}\left(\beta^{*}\right)}-1\right) i e^{2 \pi i t^{*}}\right) \\
& -\frac{\rho_{f} \omega}{a_{1}}\left[\frac{1}{\mu} \frac{J_{0}\left(K r^{*}\right)}{J_{0}(K)}+\frac{\kappa R l^{*}}{\mu} \frac{J_{1}(K)}{J_{0}(K)}-\frac{1}{\mu}\right] \psi_{s} E_{z}^{\prime} .
\end{aligned}
$$

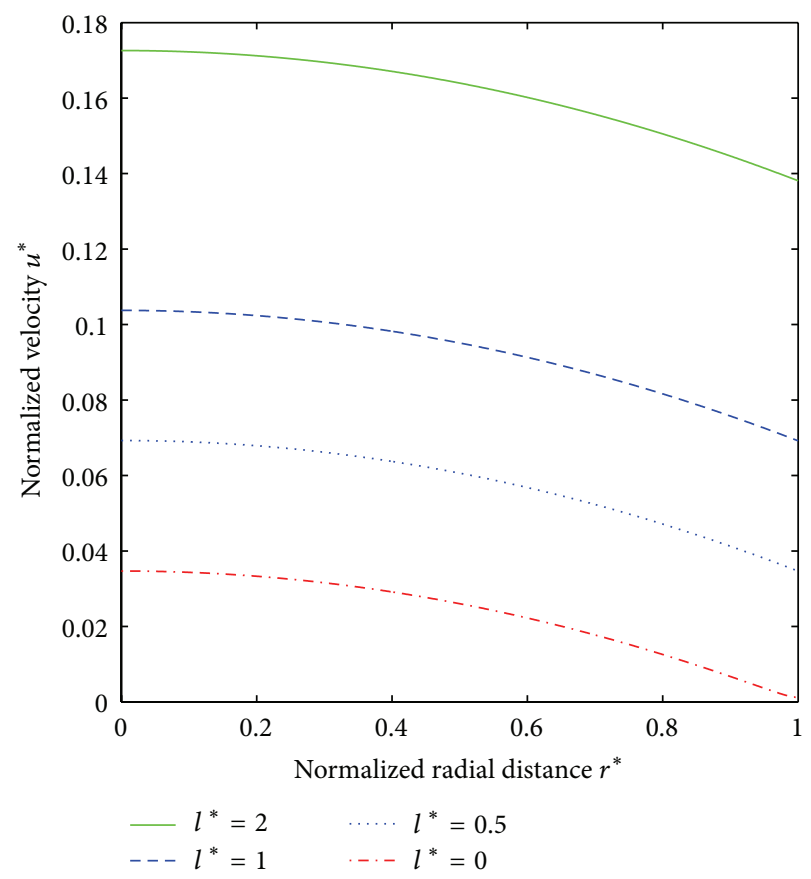

FIGURE 1: Typical velocity profile of the velocity component due to pressure gradient under different $l^{*}$ value.

Let

$$
\begin{gathered}
M=M\left(r^{*}\right)=\operatorname{Re}\left(\frac{J_{0}\left(\beta^{*} r^{*}\right)}{J_{0}\left(\beta^{*}\right)-l^{*} \beta^{*} J_{1}\left(\beta^{*}\right)}\right), \\
N=N\left(r^{*}\right)=\operatorname{Im}\left(\frac{J_{0}\left(\beta^{*} r^{*}\right)}{J_{0}\left(\beta^{*}\right)-l^{*} \beta^{*} J_{1}\left(\beta^{*}\right)}\right), \\
A_{1}=-\frac{\rho_{f} \omega}{\mu J_{0}(K)}, \quad A_{2}=\frac{\rho_{f} \omega K}{\mu J_{0}(K)} J_{1}(K) R, \\
A_{3}=\frac{\rho_{f} \omega}{\mu},
\end{gathered}
$$

then

$$
\begin{aligned}
u^{*}= & A\left(r^{*}\right) \cos \left(2 \pi t^{*}-\theta\right) \\
& +\left(A_{1} J_{0}\left(K r^{*}\right)+A_{2} l^{*}+A_{3}\right) \frac{\psi_{s} E_{z}^{\prime}}{a_{1}},
\end{aligned}
$$

where

$$
A=\sqrt{N^{2}+(1-M)^{2}}, \quad \theta=\arctan \left(\frac{M-1}{N}\right) .
$$

As the $M$ and $N$ defined above are in terms of the complex parameter $\beta^{*}$ and the Bessel functions with complex arguments, the relationship between the velocity and the slip length $l$ needs to be investigated. We first proceed to derive 


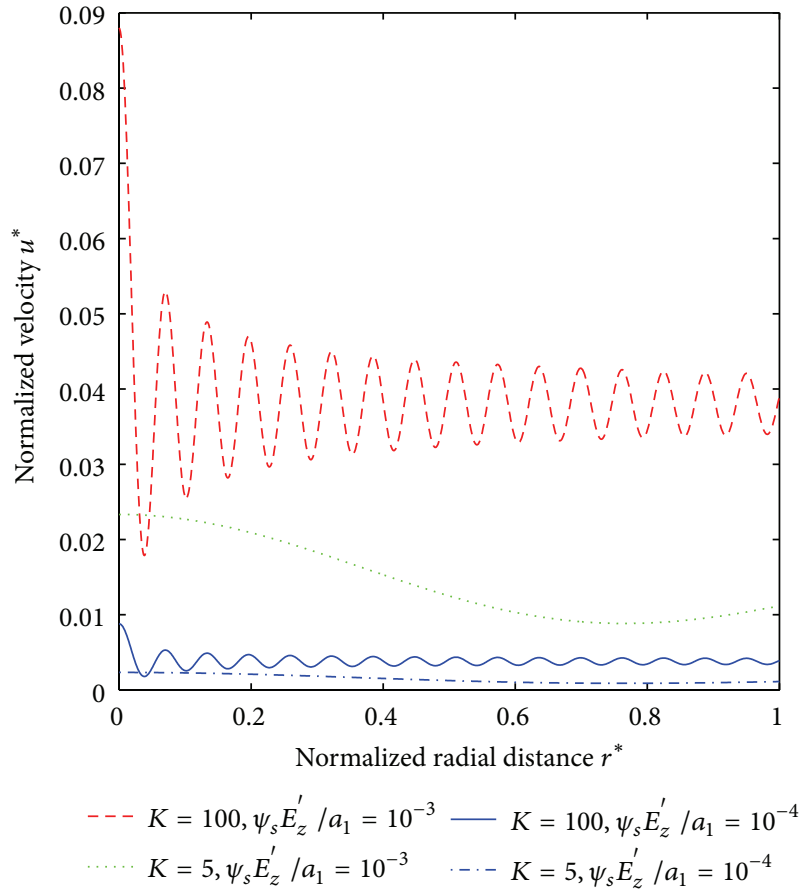

FIGURE 2: Typical profiles of the velocity component due to electric field for $l^{*}=0.5$ under different $\psi_{s} E_{z}^{\prime} / a_{1}$ and $K$ values.

more explicit formulae relating $u^{*}$ and $l$ in the real domain. As $\beta^{2}=-(\rho \omega / \mu) i=-(\rho \omega / \mu) e^{-\pi / 2}$, we get

$$
\begin{gathered}
\beta=\sqrt{\frac{\rho \omega}{2 \mu}}(1-i)=\frac{\bar{\beta}}{R}(1-i), \\
\frac{1}{\beta}=\frac{R}{2 \bar{\beta}}(1+i), \quad \beta^{*}=\bar{\beta}(1-i),
\end{gathered}
$$

where $\bar{\beta}=R \sqrt{\rho \omega / 2 \mu}$. For $\|y\| \ll 1$, then we have the following asymptotic formulae for approximating Bessel functions [12]:

$$
\begin{gathered}
J_{n}(x+y i) \approx J_{n}(x)-\frac{i y}{2}\left[J_{n+1}(x)-J_{n-1}(x)\right], \\
J_{n}(y) \simeq \frac{1}{n !}\left(\frac{y}{2}\right)^{2} .
\end{gathered}
$$

Thus, for $\bar{\beta} \ll 1$, we have

$$
\begin{gathered}
J_{0}\left(\beta^{*}\right)=J_{0}(\bar{\beta}-\bar{\beta} i) \approx J_{0}(\bar{\beta})+i \bar{\beta} J_{1}(\bar{\beta}) \approx 1+\frac{\bar{\beta}^{2}}{2} i, \\
J_{1}\left(\beta^{*}\right)=J_{1}(\bar{\beta}-\bar{\beta} i) \approx \frac{\bar{\beta}}{2}-\frac{\bar{\beta}}{2}\left[1-\frac{\bar{\beta}^{2}}{8}\right] i,
\end{gathered}
$$

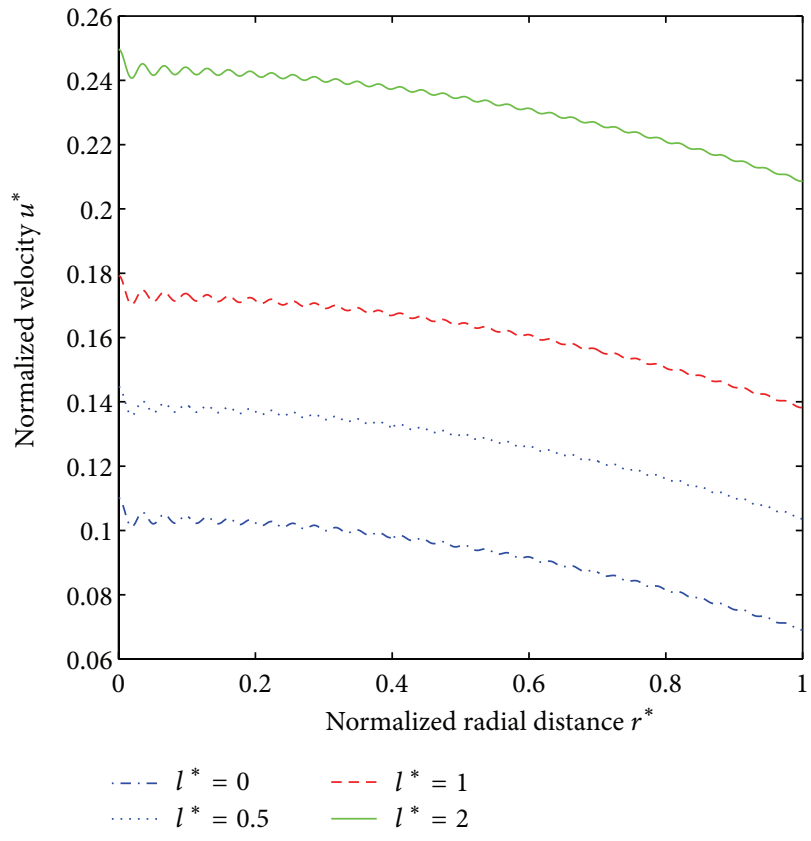

FIGURE 3: Typical velocity profile for various different values of slip length $l^{*}$ at a typical time $t^{*}=\theta / 2 \pi$, obtained with $\psi_{s} E_{z}^{\prime} / a_{1}=10^{-4}$, $K=200$.

$$
\begin{gathered}
J_{0}\left(\beta^{*} r^{*}\right) \approx 1+\frac{\bar{\beta}^{2} r^{* 2}}{2} i, \\
J_{1}\left(\beta^{*} r^{*}\right)=\frac{\bar{\beta} r^{*}}{2}-\frac{\bar{\beta} r^{*}}{2}\left[1-\frac{\left(\bar{\beta} r^{*}\right)^{2}}{8}\right] i .
\end{gathered}
$$

Substituting the above into (48) yields

$$
\begin{gathered}
M \approx \frac{1-\bar{\beta}^{4} l^{*}\left(1-8 r^{* 2}\right) / 16}{1+\bar{\beta}^{4} l^{*}\left((7 / 8)+l^{*}\right)}, \\
N \approx-\frac{\bar{\beta}^{2}\left(1-r^{* 2}+2 l^{*}\right)}{2\left[1+\bar{\beta}^{4} l^{*}\left((7 / 8)+l^{*}\right)\right]} .
\end{gathered}
$$

Hence we have

$$
\begin{aligned}
A= & A\left(r^{*}\right) \\
= & \frac{\bar{\beta}^{2}}{2\left[1+\bar{\beta}^{4} l^{*}\left((7 / 8)+l^{*}\right)\right]} \\
& \times \sqrt{\left(1-r^{* 2}+2 l^{*}\right)^{2}+\frac{\bar{\beta}^{4} l^{* 2}}{64}\left(15-8 r^{2}+16 l^{*}\right)^{2},} \\
u^{*}= & A\left(r^{*}\right) \cos \left(2 \pi t^{*}-\theta\right) \\
& +\frac{\left[A_{1} J_{0}\left(K r^{*}\right)+A_{2} l^{*}+A_{3}\right] \psi_{s} E_{z}^{\prime}}{a_{1}} .
\end{aligned}
$$

Thus, it can be concluded that the velocity consists of a time-dependent part in terms of a trigonometric function and 


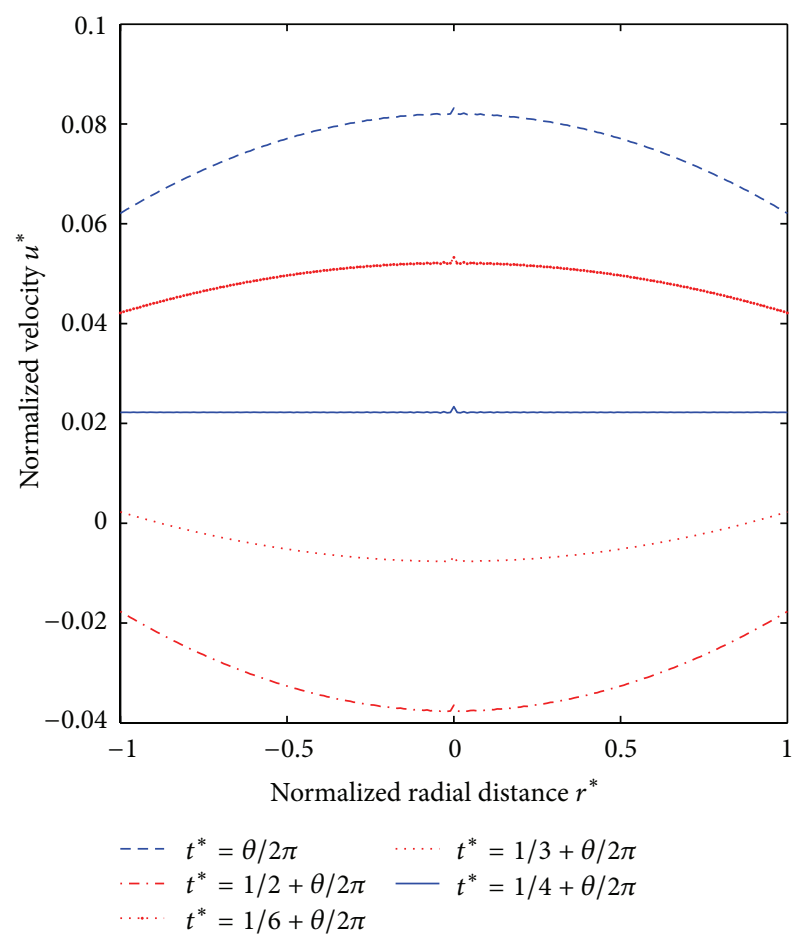

FIGURE 4: Typical velocity profile for the mixed pressure and electric driven flow at various instants of time $t^{*}$, obtained with slip length $l^{*}=1, \psi_{s} E_{z}^{\prime} / a_{1}=10^{-6}$, and $K=1500$.

a time-independent part in terms of a Bessel function of the first kind of order zero.

To demonstrate the velocity profile of the mixed pressuredriving and electrical-driving flow and the influence of the slip parameter and electric field on the flow, we carry out numerical investigation under various conditions. The typical values chosen for the model parameters $R, \rho_{f}, \omega, \mu$ are $R=$ $20 \mathrm{~mm}, \rho_{f}=1000 \mathrm{~km} / \mathrm{m}^{3}, \omega=1 / 2 \pi, \mu=0.86 \mathrm{Ns} / \mathrm{m}^{2}$. Based on [27-29], the $K$ value varies from one electrolyte solution to another; thus in this work we use a wide range of values to demonstrate the influence of $K$ on the flow. The slip length $l$ is related to the smoothness of the surface of the tubes and is controllable within certain range; hence we also use a wide range of $l$ values in the investigation to demonstrate the influence of $l$ on the flow profile and flow rate. The $\psi_{s} E_{z}^{\prime}$ is set to $0.05 \mathrm{~V}$ in all experiments except for the experiment in which the influence of the intensity of electric field relative to the pressure-driving force is to be investigated.

As the velocity profile consists of two parts due, respectively, to pressure gradient and electrical field, we first show the profile of these two velocity components in Figures 1 and 2 under different electric fields $\psi_{s} E_{z}^{\prime} / a_{1}$ and $K$ values. From the figure, it is clear that it is possible to control the velocity profile in an optimal manner based on the need of application for the mixed driving flow by finding proper combination of the pressure field and electrical field. Figures 3 and 4 show typical velocity profile for mixed driving flows.

To demonstrate the influence of the slip length on the velocity, we vary the value of $l^{*}$ from 0 to 100 while maintaining other parameter values unchanged. Figure 5 shows the

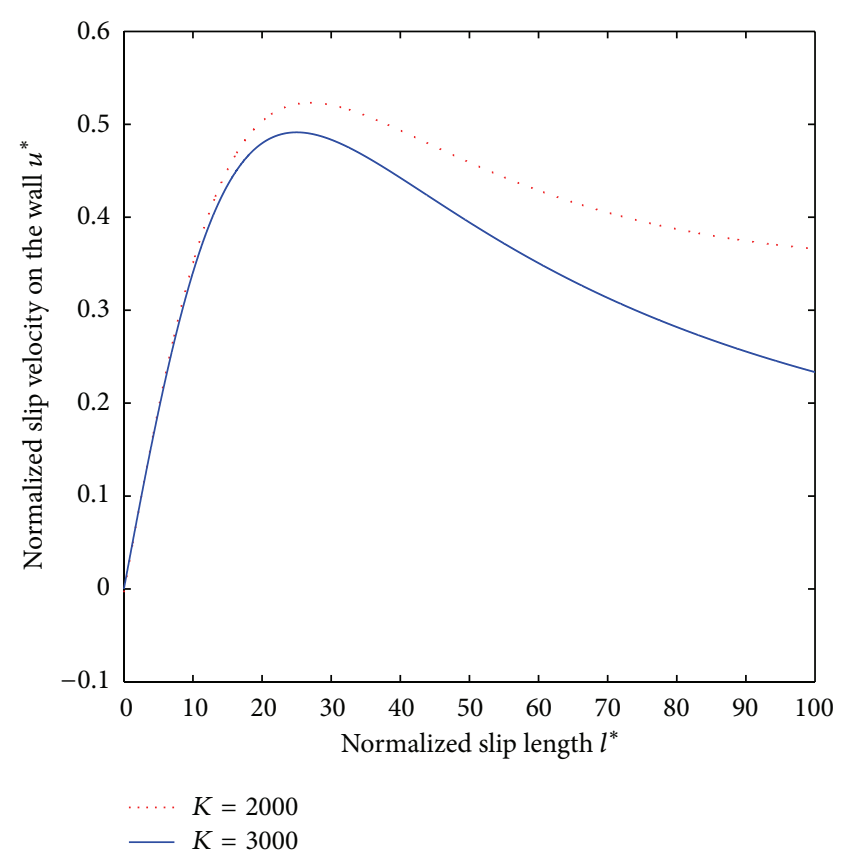

FIGURE 5: The influence of slip length on the slip velocity on the wall at a typical time $t^{*}=\theta / 2 \pi$, obtained with $\psi_{s} E_{z}^{\prime} / a_{1}=10^{-3}, l^{*}=1$.

effect of slip length on the velocity on the wall at a typical time step $t^{*}=\theta / 2 \pi$, obtained with $\psi_{s} E_{z}^{\prime} / a_{1}=10^{-6}, K=3000$, and $K=2000$. It is clear that the relationship between the slip parameter and the velocity is nonlinear and there is an optimal value for which the slip velocity takes the maximum value.

As the $K$ value changes across different electrolyte solutions, we investigate the influence of $K$ on the velocity profile. Figure 6 shows the velocity profiles for different $K$ values for $\psi_{s} E_{z}^{\prime} / a_{1}=10^{-3}$ and under the same pressure gradient and slip length $l^{*}=1$. It is clear from the result that the $K$ value influences the velocity profile significantly. For lower $K$, the velocity profile is smooth, while under high $K$ values, the velocity shows wave form profile and the frequency of the waves increases as $K$ increases. It is also noted that the amplitude of the waves increases as the $\psi_{s} E_{z}^{\prime} / a_{1}$ value increases. The magnitude of the velocity is also affected significantly by the $\psi_{s} E_{z}^{\prime} / a_{1}$ value as shown in Figure 7. As $\psi_{s} E_{z}^{\prime} / a_{1}$ increases, the magnitude of the velocity increases.

\section{Conclusions}

In this paper, an exact solution for the transient flow of an incompressible Newtonian fluid in microtubes is derived taking into account the electrokinetic effect and the boundary slip. We have shown that both boundary slip and electrokinetic field have significant effect on the flow. The results are summarized as follows.

(i) Both the pressure-gradient driving force and electrical-driving force influence the velocity profile significantly, and it is possible to construct an optimal control 


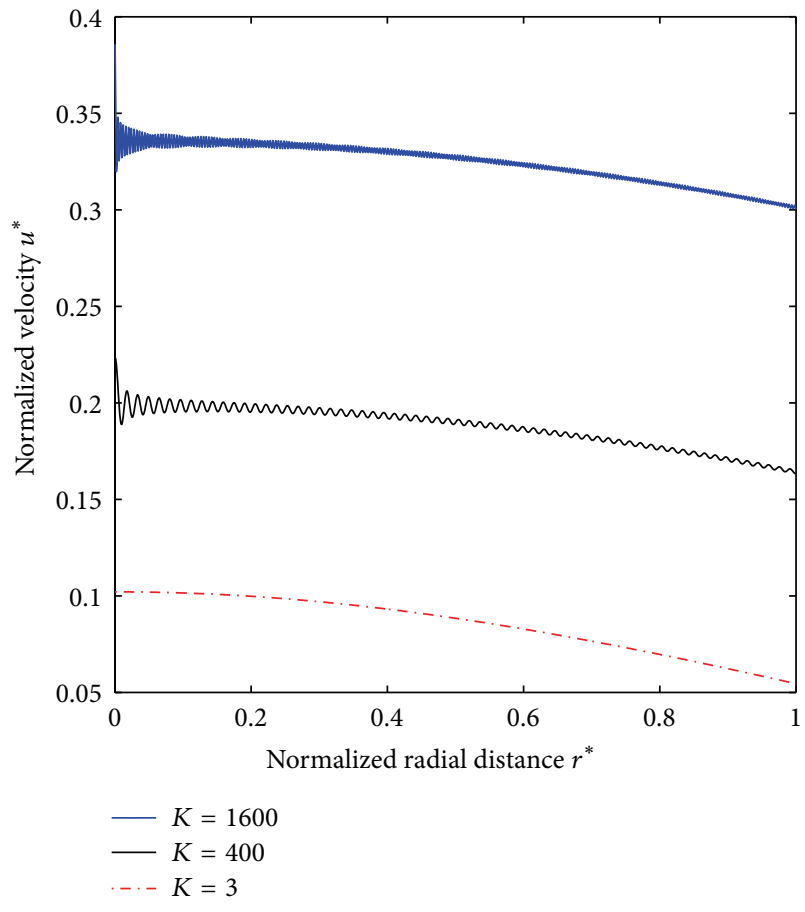

Figure 6: Influence of $K$ value on the velocity profile at a typical time $t^{*}=\theta / 2 \pi$, obtained with $\psi_{s} E_{z}^{\prime} / a_{1}=10^{-3}, l^{*}=1$.

problem to achieve the desired velocity profile based on the need of the application.

(ii) For the case of constant pressure gradient, the relationship between the slip parameter and the velocity is linear. However, for the pressure gradient in wave form, the influence of the slip parameter on the velocity magnitude is nonlinear and there exists a critical slip length where the magnitude and consequently the transient flow rate attain maximum values, which indicates that there exists an optimal slip length in terms of the magnitude of velocity and flow rate.

(iii) The $K$ value of the electrolyte solution has significant influence on the velocity profile on the cross-section of the tube. At low $K$ value, no wave form profile exists. However, as $K$ increases, the cross-section velocity exhibits wave form profile, and the frequency of the wave increases as $K$ increases.

(iv) The magnitude of the intensity of electric field relative to the pressure gradient, measured by $\psi_{s} E_{z}^{\prime} / a_{1}$, also has very significant influence on the velocity profile. As $\psi_{s} E_{z}^{\prime} / a_{1}$ increases, the velocity component increases and the amplitude of the wave in the waved form velocity profile also increases.

\section{Acknowledgments}

Qian Sun, Yonghong Wu, and Lishan Liu were supported financially by the National Natural Science Foundation of China (11071141, 11371221), the Specialized Research Foundation for the Doctoral Program of Higher Education of China (20123705110001), and the Program for Scientific Research

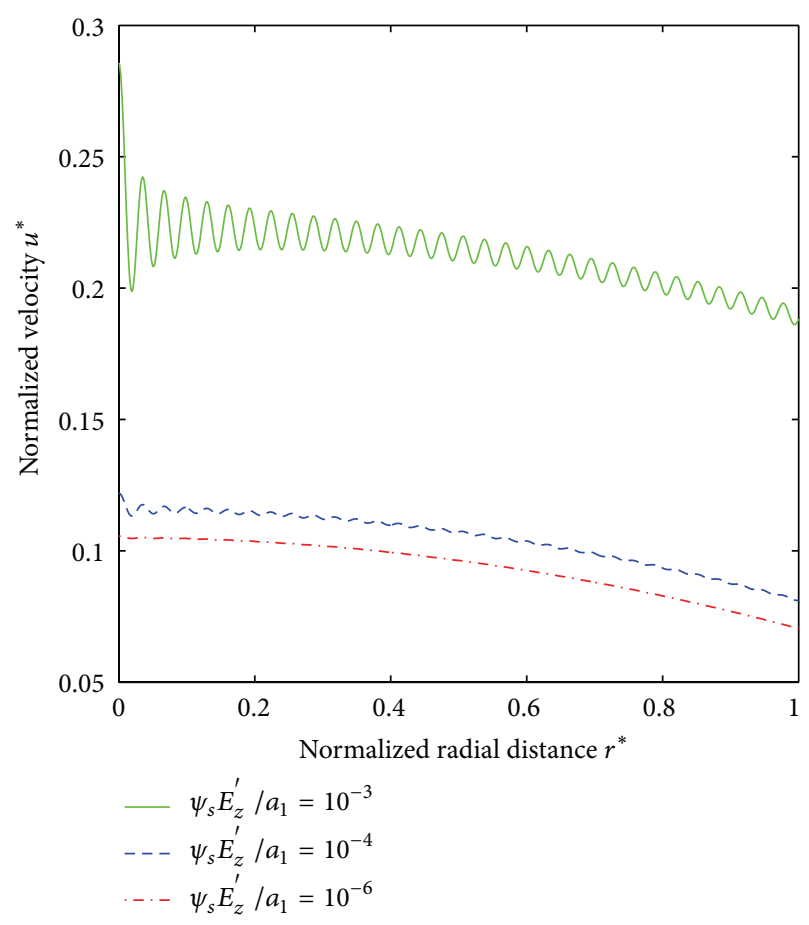

Figure 7: Influence of magnitude of electric field on the velocity profile at a typical time $t^{*}=\theta / 2 \pi, l^{*}=0.5, K=200$.

Innovation Team in Colleges and Universities of Shandong Province.

\section{References}

[1] C. E. Hunt, C. A. Desmond, D. R. Ciarlo, and W. J. Benett, "Direct bounding of micromachined silicon wafers for laser diode heat exchanger applications," Journal of Rhelogy, vol. 1, no. 26, pp. 152-156, 1991.

[2] Y. J. Song and T. S. Zhao, "Modelling and testing of a thermallydriven phase-change nonmechanical micropump," Journal of Micromechanics and Microengineering, vol. 11, no. 6, pp. 713-719, 2001.

[3] D. J. Harrison, K. Fluri, K. Seiler, Z. Fan, C. S. Effenhauser, and A. Manz, "Micromachining a miniaturized capillary electrophoresis-based chemical analysis system on a chip," Science, vol. 261, no. 5123, pp. 895-897, 1993.

[4] Y.-C. Su and L. Lin, "A water-powered micro drug delivery system," Journal of Microelectromechanical Systems, vol. 13, no. 1, pp. 75-82, 2004.

[5] J. J. Nakane, M. Akeson, and A. Marziali, "Nanopores sensors for nucleic acid analysis," Journal of Physics, vol. 15, no. 32, p. R1363, 2005.

[6] J. C. Slattery, Advanced Transport Phenomena, Cambridge University Press, 1999.

[7] Y. H. Wu, B. Wiwatanapataphee, and X. Yu, "An enthalpy control volume method for transient mass and heat transport with solidification," International Journal of Computational Fluid Dynamics, vol. 18, no. 7, pp. 577-584, 2004.

[8] B.-Y. Cao, M. Chen, and Z.-Y. Guo, "Liquid flow in surfacenanostructured channels studied by molecular dynamics simulation," Physical Review E, vol. 74, no. 6, Article ID 066311, 2006. 
[9] B.-Y. Cao, M. Chen, and Z.-Y. Guo, "Velocity slip of liquid flow in nanochannels," Acta Physica Sinica, vol. 55, no. 10, pp. 53055310, 2006.

[10] L. Szalmas, "Slip-flow boundary condition for straight walls in the lattice boltzmann model-art," Physical Review, vol. 6, p. 6710, 2006.

[11] J. Xu and Y. Li, "Boundary conditions at the solid-liquid surface over the multiscale channel size from nanometer to micron," International Journal of Heat and Mass Transfer, vol. 50, no. 1314, pp. 2571-2581, 2007.

[12] Y. Zhu and S. Granick, "Rate-dependent slip of Newtonian liquid at smooth surfaces," Physical Review Letters, vol. 87, no. 9, Article ID 096105, 4 pages, 2001.

[13] P. Huang and K. S. Breuer, "Direct measurement of slip length in electrolyte solutions," Physics of Fluids, vol. 19, no. 2, Article ID 028104, 2007.

[14] R. Pit, H. Hervet, and L. Léger, "Direct experimental evidence of slip in hexadecane: solid interfaces," Physical Review Letters, vol. 85, no. 5, pp. 980-983, 2000.

[15] Y. Christophe, B. Catherine, and C. B. Ccile, "Joseph pierre, achieving large slip with superhydrophobic surfaces:scaling laws for generic geometries," Physics of Fluids, vol. 19, pp. 123130, 2007.

[16] B. Wiwatanapataphee, D. Poltem, Y. H. Wu, and Y. Lenbury, "Simulation of pulsatile flow of blood in stenosed coronary artery bypass with graft," Mathematical Biosciences and Engineering, vol. 3, no. 2, pp. 371-383, 2006.

[17] B. Wiwatanapataphee, Y. H. Wu, J. Archapitak, and P. F. Siew, "A numerical study of the turbulent flow of molten steel in a domain with a phase-change boundary," Journal of Computational and Applied Mathematics, vol. 166, no. 1, pp. 307-309, 2004.

[18] Y. H. Wu and B. Wiwatanapataphee, "Modelling of turbulent flow and multi-phase heat transfer under electromagnetic force," Discrete and Continuous Dynamical Systems B, vol. 8, no. 3, pp. 695-706, 2007.

[19] M. T. Matthews and J. M. Hill, "Newtonian flow with nonlinear navier boundary condition," Acta Mechanica, vol. 191, no. 3, pp. 195-217, 2007.

[20] S.-P. Yang and K.-Q. Zhu, "Analytical solutions for squeeze flow of Bingham fluid with Navier slip condition," Journal of NonNewtonian Fluid Mechanics, vol. 138, no. 2-3, pp. 173-180, 2006.

[21] C. L. Rice and R. Whitehead, "Electrokinetic flow in a narrow cylindrical capillary," Journal of Physical Chemistry, vol. 69, no. 11, pp. 4017-4024, 1965.

[22] S. Levine, J. R. Marriott, and K. Robinson, "Theory of electrokinetic flow in a narrow parallel-plate channel," Journal of the Chemical Society, vol. 71, pp. 1-11, 1975.

[23] C. Yang, D. Li, and J. H. Masliyah, "Modeling forced liquid convection in rectangular microchannels with electrokinetic effects," International Journal of Heat and Mass Transfer, vol. 41, no. 24, pp. 4229-4249, 1998.

[24] G. M. Mala, D. Q. Li, and J. D. Dale, "Heat transfer and fluid flow in microchannels," International Journal of Heat and Mass Transfer, vol. 40, pp. 3079-3088, 1997.

[25] Y. H. Wu, B. Wiwatanapataphee, and M. Hu, "Pressure-driven transient flows of Newtonian fluids through microtubes with slip boundary," Physica A, vol. 387, no. 24, pp. 5979-5990, 2008.

[26] J. Yang and D. Y. Kwok, "Analytical treatment of flow in infinitely extended circular microchannels and the effect of slippage to increase flow efficiency," Journal of Micromechanics and Microengineering, vol. 13, no. 1, pp. 115-123, 2003.
[27] P. C. H. Li and J. D. Harrison, "Transport manipulation and reaction of biological cells on chip using electrokinetic effects," Analytical Chemistry, vol. 69, no. 8, pp. 1564-1568, 1997.

[28] Z. H. Fan and D. J. Harrison, "Micromachining of capillary electrophoresis injectors and separators on glass chips and evaluation of flow at capillary intersections," Analytical Chemistry, vol. 66, no. 1, pp. 177-184, 1994.

[29] G. Ocvirk, M. Munroe, T. Tang, R. Oleschuk, K. Westra, and D. J. Harrison, "Electrokinetic control of fluid flow in native poly(dimethylsiloxane) capillary electrophoresis devices," Electrophoresis, vol. 21, no. 1, pp. 107-115, 2000. 


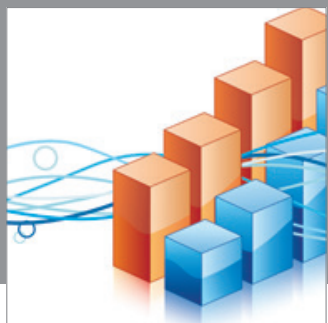

Advances in

Operations Research

mansans

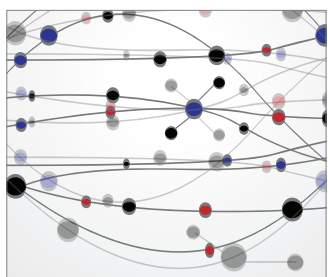

The Scientific World Journal
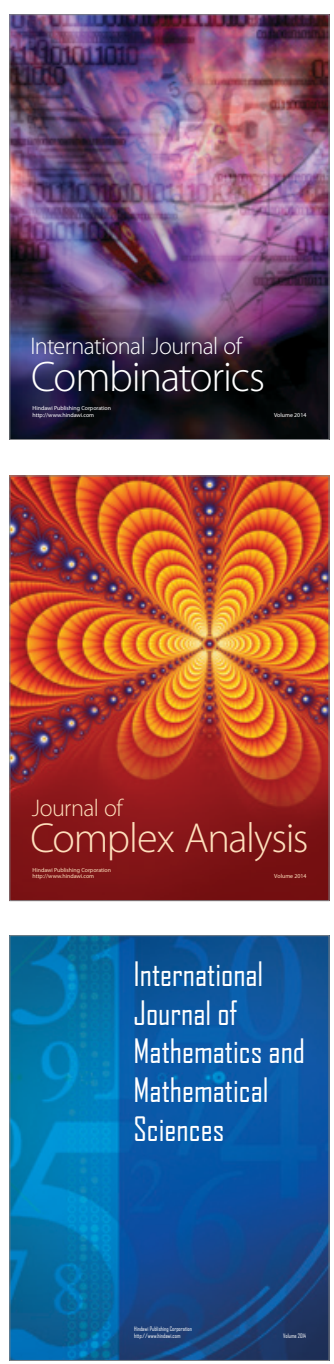
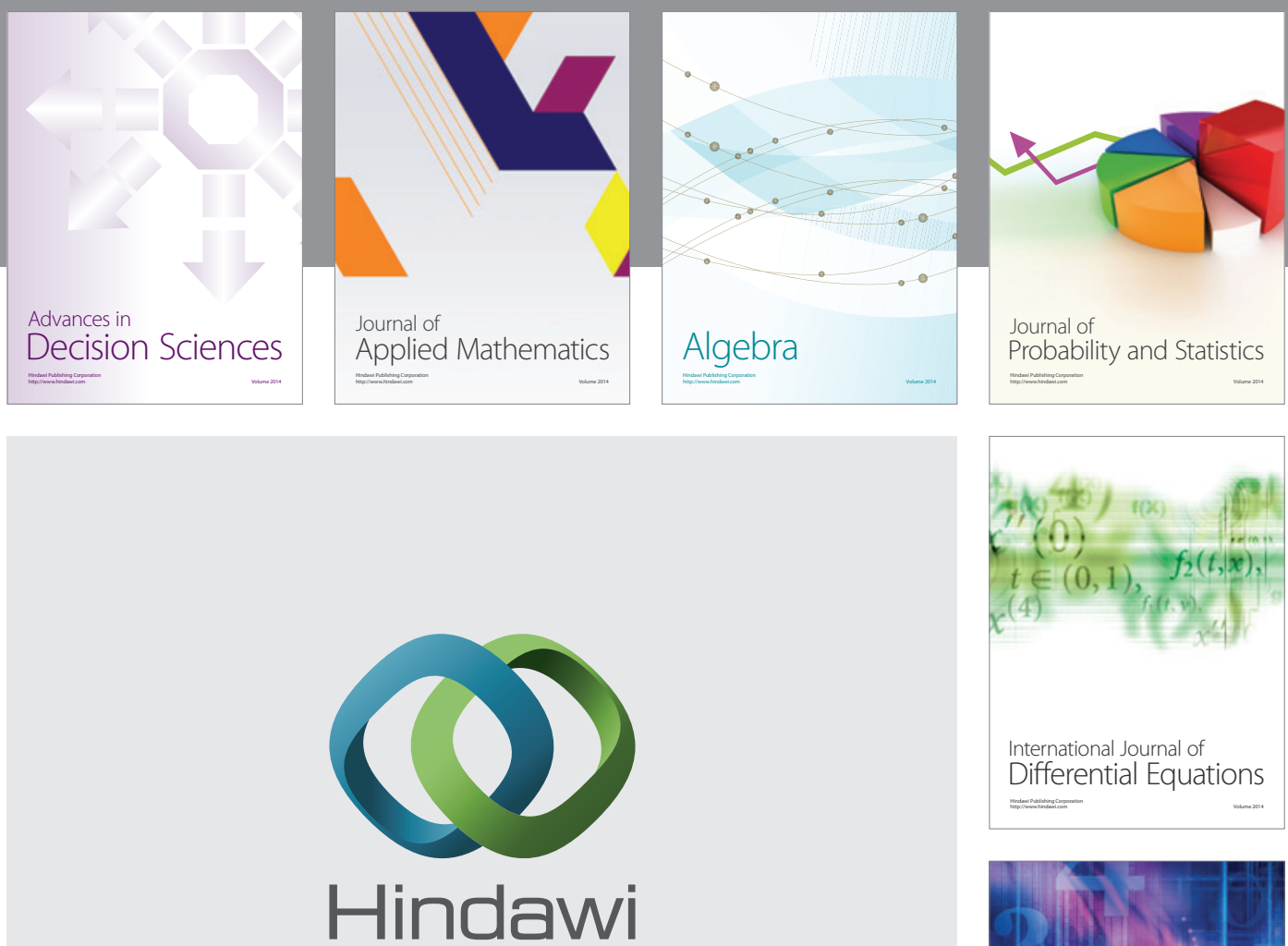

Submit your manuscripts at http://www.hindawi.com
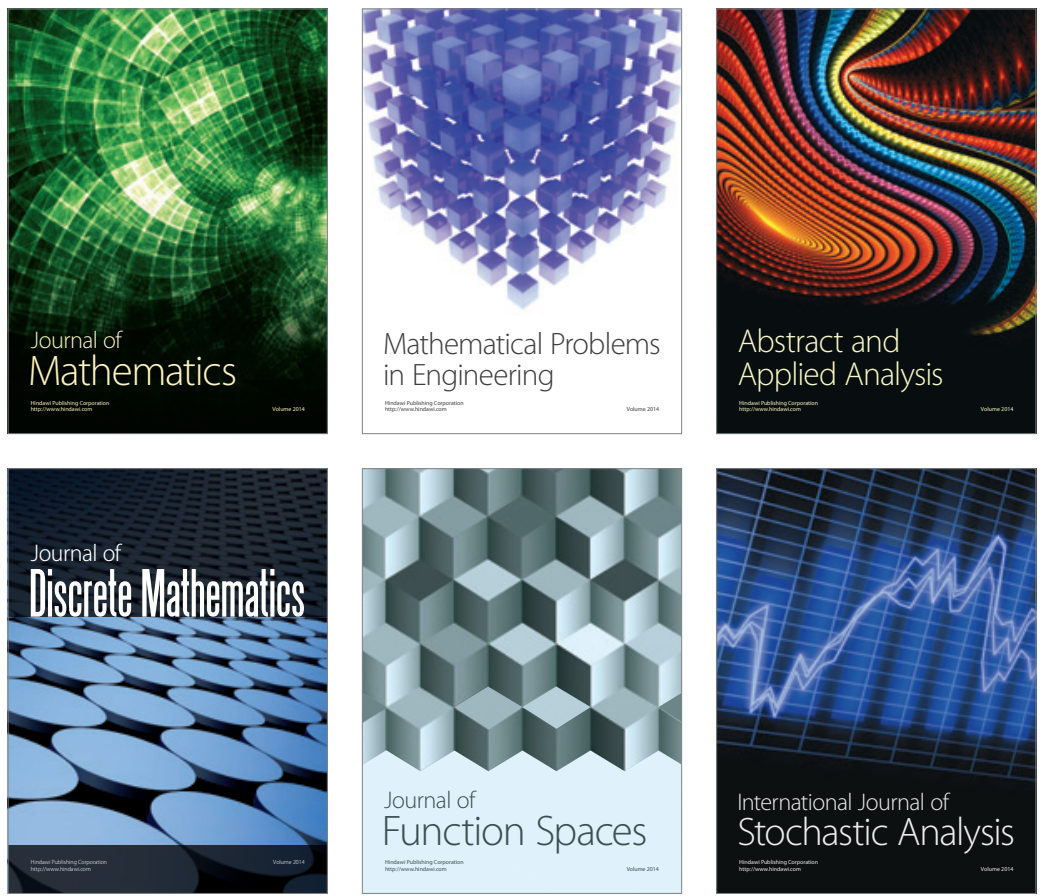

Journal of

Function Spaces

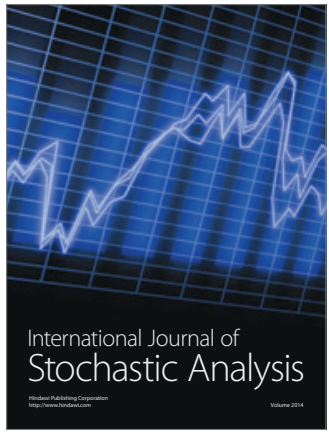

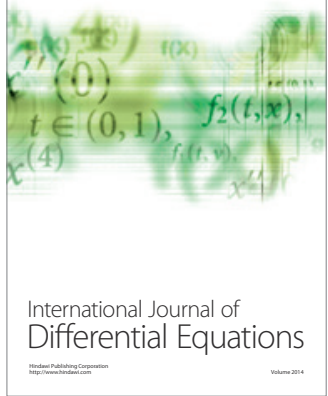
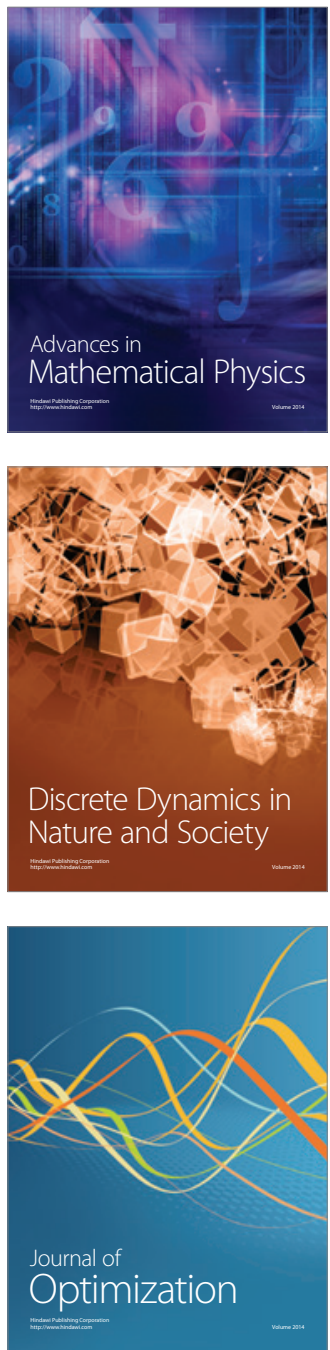